\title{
Geospatial-temporal Evolution of urbanization level in Henan Province(2011-2015)
}

\author{
ZHANG Kaiguang ${ }^{1, a^{*}}$, BA Mingting ${ }^{2, b}$ and MENG Hongling ${ }^{3, c}$ \\ ${ }^{1}$ Institute of 3S Technology, Zhengzhou Normal University, Zhengzhou 450044,China \\ azzgis@sina.com, bbmt1234@126.com, chnmhl@126.coml
}

\begin{abstract}
Keywords: urbanization level, spatial relationship, spatial statistical analysis, spatial correlation Abstract. The best is to read these instructions and follow the outline of this text. Urbanization is the process of regional population structure adjustment caused by productivity development, scientific and technological progress and industrial structure adjustment, which is also the inevitable result of the regional interaction. In the paper, the spatial relationships among cities in a region are designed by using the inverse of the shortest time distance between cities in express traffic system, the geospatial-temporal characteristics and evolution patterns of urbanization levels are studied by using spatial statistical analysis methods. The results show that, in Henan province, the differences of urbanization levels are significant, the urbanization levels of cities in the northern part are higher than that of cities in the southern part, which gradually reduce along the direction from southeast to northwest. From 2011 to 2015, the province's urbanization levels have been significantly improved, the city rankings remain unchanged, as the urbanization levels of cities rapid growth, the differences among them are gradually reduced. Urbanization level growth and urbanization level is inversely proportional. There are significantly aggregation characteristics in the spatial distribution of urbanization levels, showing a high-high aggregation in the northwestern part, and low-low aggregation in the southeast part, and from 2011 to 2015, the aggregation tendency gradually stronger.
\end{abstract}

\section{Introduction}

Urbanization is the transformation process of traditional agricultural society to non-agricultural society as industrial society and services society, along with productivity development, scientific and technological progress and industrial structure adjustment, is also the inevitable trend of social development [1-4]. In recent years, researches on urbanization mainly focus on the characteristics of cities and factors of urbanizing, make some valuable results [5-11]. However, the influence factors of urbanization studies mainly base on the influence factors of local economy development, society, geography and environment itself, ignoring the interaction between cities. Some economic geography phenomenon or an attribute value in one regional spatial unit is related with adjacent regional space unit on the same phenomenon or attribute value. The interactions among regions in an area, especially traffic distribution, also plays an important role on the urbanization levels [2,6,8,11].

Henan Province is located in the middle of China, on the middle and lower reaches of Yellow River, is agricultural and populous province in China. The rationality of national economic structure lag behind the country's in some areas. In 2015, the province's agricultural output value accounted for more than $11.9 \%$ higher than the national average of 2.74 percent, secondary industry output value accounted for more than $51 \%$ higher than the national average of 8.36 percent, the tertiary industry output value accounted for $37.1 \%$ lower than the national average of 11.10 percent, urbanization level 45.2\% lower than the national level of 10.9 percent [12].

A major agricultural province and a large number of rural population are the main reason for holding back of urbanization level, but too concentrated industrial layout, relatively poor traffic infrastructure as well as undeveloped tertiary industry, and the resulting regional differences in the urbanization levels are also important factor [6,9].

Based on the inverse of the shortest time distance between cities in express traffic system as the spatial relation, this paper uses spatial statistical analysis methods to study the geospatial-temporal characteristics and evolution patterns of urbanization levels, in order to correctly understand the 
spatial distribution characteristics of urbanization levels, then formulate coordinated regional development policy provide a useful reference. Research data are mainly from Henan Statistical Yearbook (2011-2015), the spatial data are derived from Henan basic spatial database and Henan traffic information database.

\section{Research methods}

Iterative clustering analysis. Spatial cluster analysis is an important tool for spatial statistical analysis, which divides the samples into different classes with similar attributes according to the similarity degree $C_{i j}$ between samples and the distance $D_{i j}$ between classes.

$$
C_{i j}=\sum_{i=1}^{n}\left(x_{i i}-\overline{x_{i}}\right)\left(x_{i j}-\overline{x_{j}}\right) / \sigma_{i} \sigma_{j} ; D_{i j}=\sum_{k=1}^{n} \sum_{t=1}^{n}\left(x_{k i}-\overline{x_{i}}\right)\left(x_{i j}-\overline{x_{j}}\right)
$$

where $x_{i}, \overline{x_{i}},(n-1) \sigma_{i}$ are sample value, sample mean and sample standard deviation respectively. Iterative clustering analysis, analyzes a continuous sample observation through the sample sequence step and truncating, is an important method to observe the sample unit level and trends in the sequence [12].

Spatial weights matrix. Spatial weights matrix $W=\left(w_{i j}\right)_{n n}$ is a two-dimensional matrix, $w_{i j}$ representing the influence level of unit $j$ to unit $i$ in a region. Sometimes there is $w_{i j} \neq w_{j i}$ in non-Euclidean distance space.

Global spatial autocorrelation. Global spatial autocorrelation index Moran I, describes the spatial difference and spatial similarity level among all adjacent regions for samples with some regular distribution.

$$
I=\sum_{i=1}^{n} w_{i j}\left(x_{i}-\bar{x}\right)\left(x_{j}-\bar{x}\right) / s^{2} \sum_{i=1}^{n} \sum_{j=1}^{n} w_{i j}
$$

\begin{tabular}{|c|c|c|c|c|c|c|c|c|c|c|c|}
\hline & $\mathrm{ZZ}$ & $\mathrm{KF}$ & LY & PDS & $A Y$ & HB & XX & $\mathrm{JZ}$ & PY & $\mathrm{XC}$ & LH \\
\hline 011 & 6 & 5.0 & 4.33 & 41.39 & 8.60 & 8. & 41. & 47 & 6 & 39.10 & 39. \\
\hline 012 & 64.82 & 37.75 & 46.13 & 43.14 & & 49.76 & 42.89 & o & & 40.92 & 0 . \\
\hline 013 & .2 & .7 & 47.93 & 44.97 & 42 & 51.56 & 44.69 & 50.72 & 0 & 42.83 & 42.84 \\
\hline 17 & 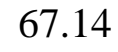 & 41.08 & .40 & 46.37 & 43 & 5 & 45.95 & 14 & 36.87 & 44.19 & נוד \\
\hline \multirow[t]{2}{*}{2015} & 68.34 & 42.64 & 50.90 & 47.78 & 45.19 & 53.75 & 47.64 & 53.41 & 38.61 & 45.60 & 45.77 \\
\hline & NY & SQ & XY & SMX & $\mathrm{ZK}$ & ZMD & JY & Var & Range & $\mathrm{HN}$ & China \\
\hline 011 & 33.00 & 29.75 & 34.36 & 44.25 & 29.73 & 29.75 & 49.44 & 75.86 & 33.89 & 38.82 & 49.68 \\
\hline 012 & 34.85 & 31.54 & 36.25 & 46.00 & 31.50 & 31.54 & 51.44 & 74.20 & 33.32 & 40.57 & 51.27 \\
\hline 013 & 36.81 & 33.49 & 38.19 & 47.60 & 33.44 & 33.44 & 53.44 & 72.67 & 32.84 & 42.43 & 52.57 \\
\hline 2014 & 38.26 & 35.03 & 39.66 & 49.11 & 34.85 & 34.93 & 54.17 & 69.82 & 32.29 & 43.80 & 53.73 \\
\hline 2015 & 39.54 & 36.50 & 41.03 & 50.22 & 36.25 & 36.36 & 56.94 & 69.93 & 32.09 & 45.20 & 54.77 \\
\hline
\end{tabular}

Table 1 Urbanization levels in Henan province

Local spatial autocorrelation index Local Moran I, describes the spatial pattern on different spatial locations, reflects the aggregation and difference characteristics for one region with some significant similarity value between adjacent regions.

$$
\boldsymbol{I}_{i}=\left(x_{i}-\bar{x}\right) \sum_{i=1, i j j}^{n} w_{i j}\left(x_{j}-\bar{x}\right) / s^{2} \sum_{i=1}^{n} \sum_{j=1}^{n} w_{i j}
$$

Where $x_{i} 、 \overline{x_{i}} 、(n-1) \sigma_{i}$ are sample value, sample mean and sample standard deviation respectively, $\boldsymbol{w}_{i j}$ is the spatial weights unit $j$ to unit $i$. Under the significance level $\alpha=0.05, Z$-value reflects the correlation degree. 


\section{Geospatial-temporal distribution and its Evolution pattern of urbanization levels in Henan province}

Digital characteristics of urbanization levels in Henan Province. From 2011 to 2015, the province's urbanization levels continue to accelerate, the gap with national average levels gradually decrease (Table 1). In 2011, the urbanization levels of 11 cities are higher than the provincial average, one city higher than the national average. In 2012 and 2015, 10 cities are higher than the provincial average, two cities reach or exceed the national average. In 2013 and 2014, higher than the provincial average have 11 cities, Zhengzhou and Jiyuan are above the national average of 2.17 and 13.57 percent respectively. In the 5 years, the city's urbanization level rankings remain unchanged, but the range and variance decreases, indicating that as the urbanization levels in the province's rapid growing, the differences between cities is gradually reduced.

In the view of geospatial distribution (Fig. 1), for 5 years, the distributions of province's urbanization levels are substantially similar, the urbanization level of Zhengzhou, provincial capital, has maintained a leading position, the urbanization levels of cities in the northern part are higher than that of cities in southern part, and along the direction of northwest to the southeast decreases.

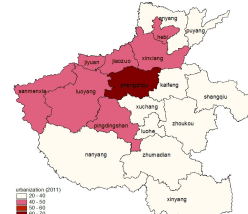

(a) 2011

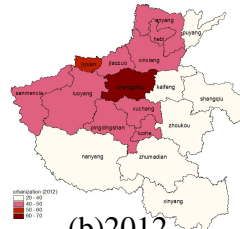

(b) 2012

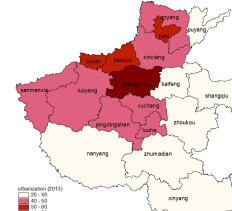

(c) 2013

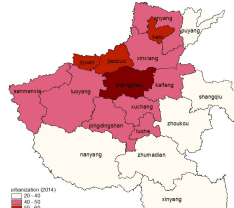

(d) 2014

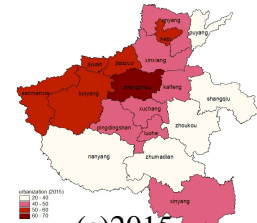

(e) 2015

Fig.1 the spatial distribution of urbanization levels in Henan province

Classification characteristic of urbanization levels in Henan Province. In the Euclidean distance between samples and the sum of squares of deviations, using iterative clustering analysis method on the urbanization levels in the 5 years, the urbanization levels of all cities in Henan province, by all combinations of step and truncating, will always be divided into high, medium and low levels (Fig. 2 and Table 2), the samples in each class remain unchanged. Zhengzhou becomes independent one class, the second class contains Luoyang, Pingdingshan, Anyang, Hebi, Xinxiang, Jiaozuo, Xuchang, Luohe, Sanmenxia, Jiyuan, the third class includes Kaifeng, Puyang, Nanyang, Shangqiu, Xinyang, Zhoukou, Zhumadian. From the geospatial distribution, the second class distributes in the central

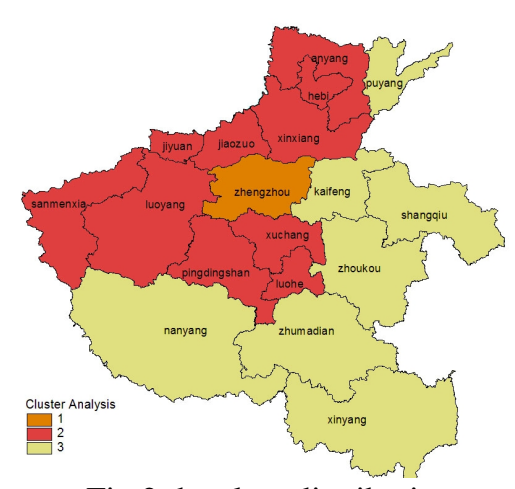

Fig.2 the class distribution and northwestern parts, while the third class distributes in the eastern and southeastern parts. As the same trend of province's urbanization level, the weighted average of all classes is gradually increasing, but the growth rates are significant differences, and the growth rates are inversely related to the urbanization level.

Table 2 The cluster analysis of urbanization levels in Henan province

\begin{tabular}{llllccl}
\hline & 2011 & 2012 & 2013 & 2014 & 2015 & Number \\
\hline I center & 63.62 & 64.82 & 66.28 & 67.14 & 68.34 & ZZ \\
II center & 48.01 & 49.76 & 51.56 & 52.8 & 53.75 & LY,PDS,AN'HB,XX,JZ,XC,LH,SM \\
& & & & & $3, J Y$ & X,JY \\
III center & 29.73 & 31.50 & 33.44 & 34.85 & 36.25 & KF,PY,NY,SQ,XY,ZK,ZMD \\
\hline
\end{tabular}

spatial autocorrelation analysis in Henan Province. Spatial weights matrix.In the process of structural adjustment of regional population, regional urbanization level is affected not only by the internal social productivity development, application level of science and technology and industry structure, the interactions among cities in the region is also important factors. Economically developed regions attract more migrants, resulting in rapid increase in the level of urbanization. Population migration between regions, not only by the limitations of traditional living habits, but also 
influenced by factors such as communications and transportation between regions, good communication and convenient transportation will greatly enhance the attractiveness of a city, but the magnitude of the attraction with the distance far gradually weakened [7,12].

The weight of city $j$ to city $i$, reflects the influence of $j$ to city $i$, defined as the inverse of the shortest time distance between $j$ and $i$ in express traffic system

$$
w_{i j}=\sum_{k=1}^{m} \frac{1}{T_{k}},
$$

Where $m$ is the number of routes with the traveling time less than the time threshold defined before. The larger $w_{i j}$ means the more attractiveness of population migration, the higher influence on the increase of urbanization level of $i$. Using Eq.4, based on Henan express traffic system and the traffic data corresponding to the each year, the spatial weights matrices are calculated, the result for 2015 is showed in Table $3[13,14]$.

Table 3 The spatial Weight matrix in Henan province(2015)

\begin{tabular}{|lllllllllllllllllll}
\hline & ZZ & KF & LY & PDS AY & HB & XX & JZ & PY & XC & LH & SMXNY & SQ & XY & ZK & ZMD JY \\
\hline ZZ & 0 & 5.8 & 4.9 & 2.2 & 0 & 0 & 3.7 & 5.6 & 0 & 4.9 & 0 & 0 & 0 & 0 & 0 & 2 & 0 & 1.5 \\
KF & 5.8 & 0 & 0 & 0 & 0 & 0 & 3 & 0 & 1.4 & 0 & 0 & 0 & 0 & 3.8 & 0 & 2.2 & 0 & 0 \\
LY & 4.9 & 0 & 0 & 3.4 & 0 & 0 & 0 & 2.9 & 0 & 1.8 & 0.8 & 2 & 0.4 & 0 & 0 & 0 & 0 & 4.2 \\
PDS & 2.2 & 0 & 3.4 & 0 & 0 & 0 & 0 & 0 & 0 & 3.5 & 1.7 & 0 & 2.3 & 0 & 0.7 & 0 & 0 & 0 \\
AY & 0 & 0 & 0 & 0 & 0 & 4 & 0 & 0 & 3.4 & 0 & 0 & 0 & 0 & 0 & 0 & 0 & 0 & 0 \\
HB & 0 & 0 & 0 & 0 & 4 & 0 & 3.1 & 0 & 2.4 & 0 & 0 & 0 & 0 & 0 & 0 & 0 & 0 & 0 \\
XX & 3.7 & 3 & 0 & 0 & 0 & 3.1 & 0 & 3.3 & 1.3 & 0 & 0 & 0 & 0 & 0 & 0 & 0 & 0 & 0 \\
JZ & 5.6 & 0 & 2.9 & 0 & 0 & 0 & 3.3 & 0 & 0 & 0 & 0 & 0 & 0 & 0 & 0 & 0 & 0 & 3 \\
PY & 0 & 1.4 & 0 & 0 & 3.4 & 2.4 & 1.3 & 0 & 0 & 0 & 0 & 0 & 0 & 0 & 0 & 0 & 0 & 0 \\
XC & 4.9 & 0 & 1.8 & 3.5 & 0 & 0 & 0 & 0 & 0 & 0 & 2.7 & 0 & 0 & 1.8 & 0.7 & 4.7 & 0 & 0 \\
LH & 0 & 0 & 0.8 & 1.7 & 0 & 0 & 0 & 0 & 0 & 2.7 & 0 & 0 & 1 & 0 & 0 & 3 & 2.2 & 0 \\
SM & 0 & 0 & 2 & 0 & 0 & 0 & 0 & 0 & 0 & 0 & 0 & 0 & 0.5 & 0 & 0 & 0 & 0 & 0 \\
NY & 0 & 0 & 0.4 & 2.3 & 0 & 0 & 0 & 0 & 0 & 0 & 1 & 0.5 & 0 & 0 & 0.9 & 0 & 0.8 & 0 \\
SQ & 0 & 3.8 & 0 & 0 & 0 & 0 & 0 & 0 & 0 & 1.8 & 0 & 0 & 0 & 0 & 0 & 2.3 & 0 & 0 \\
XY & 0 & 0 & 0 & 0.7 & 0 & 0 & 0 & 0 & 0 & 0.7 & 0 & 0 & 0.9 & 0 & 0 & 0.6 & 1.7 & 0 \\
ZK & 2 & 2.2 & 0 & 0 & 0 & 0 & 0 & 0 & 0 & 4.7 & 3 & 0 & 0 & 2.3 & 0.6 & 0 & 1 & 0 \\
ZM & 0 & 0 & 0 & 0 & 0 & 0 & 0 & 0 & 0 & 0 & 2.2 & 0 & 0.8 & 0 & 1.7 & 1 & 0 & 0 \\
JY & 1.5 & 0 & 4.2 & 0 & 0 & 0 & 0 & 3 & 0 & 0 & 0 & 0 & 0 & 0 & 0 & 0 & 0 & 0 \\
\hline
\end{tabular}

Global spatial autocorrelation analysis. Using global spatial autocorrelation analysis on the spatial distribution of urbanization levels (2011-2015) in Henan province, the results are listed in Table4, all Moran indexes are positive, and all Z-values are almost 2 times of the standard threshold (1.96), all of these indicate that the distribution of urbanization levels have some significant spatial positive correlation characteristics, that is, the cities with higher (lower) urbanization levels tend to aggregating in space. The increasing Z-values year by year indicate that the province's urbanization levels have been developing a distinctly aggregating region, the spatial distribution patterns have been becoming clear.

Table 4 The Moran I indexes in Henan province(2011-2015)

\begin{tabular}{llllll}
\hline & 2011 & 2012 & 2013 & 2014 & 2015 \\
\hline Moran I & 0.283 & 0.2886 & 0.2917 & 0.2922 & 0.3003 \\
Z-value & 3.7465 & 3.7483 & 3.8214 & 3.9852 & 4.0184
\end{tabular}

Local spatial autocorrelation analysis. Carry out local spatial autocorrelation analysis on urbanization levels (2011-2015) in Henan province, under the significant level (0.05), the geospatial distributions of urbanization levels have the same spatial characteristics (Fig.3). All of these show 
that the differences of urbanization levels among cities in the province remain unchanged, but gradually obvious.

Combined with economic growth data, we can see that the cities with higher level of urbanization have relatively developed economy, and more attractiveness of population migration from undeveloped economic regions, thereby greatly enhancing the urbanization level.

\section{Conclusions}

This paper utilizes the geospatial statistical methods to study the urbanization levels of cities in Henan province(2011-2015), describes the province's urbanization levels, the characteristics of spatial distribution, and evolution pattern. The results show that

i)The differences of urbanization levels are significant, the levels of cities in the northern part are higher than that of cities in the southern part, which gradually reduce along the direction from southeast to northwest. The regions with low urbanization levels have relatively undeveloped economy and relatively high population density.

ii)From 2011 to 2015, the province's urbanization levels have been significantly improved, the city rankings remain unchanged, as the urbanization levels of cities rapid growth, the differences

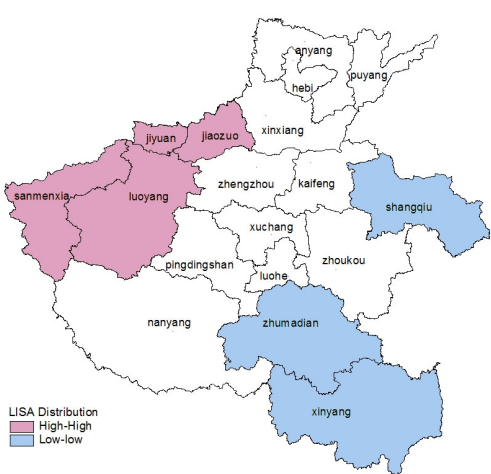

Fig.3 LISA distribution among cities are gradually reduced.

iii)The urbanization levels of cities in the province can be divided into high, medium and low. In the 5 years, the level structures have not changed, the growth rates have obvious difference, and the urbanization levels are inversely related to their growth rates.

iv)There are significantly aggregation characteristics in the spatial distribution of urbanization levels, showing a high-high aggregation in the northwestern part, and low-low aggregation in the southeast part, and from 2011 to 2015, aggregation tendency gradually stronger.

\section{Reference}

[1] Yao Shimou, Zhang Pingyu. The theory and practice of new urbanization in China. ,Scientia Geographica Sinica, 2014,34(4), p.641-647.

[2] Xiao Kuixi, Yang Yan, Liu Hui. Study on enhancing radiation of new york and the reference for guangzhou .Urban Insight, 2013,(5), p.50 56.

[3] Lin Xueqin, Wang Dai, Ren Wangbing. Research on the mechanism of urbanization to economic increase in China. Geographical Research, 2013,32(4), p.691-700.

[4] Li Qiang, Chen Yulin, Li Jingming. On the development moe of Chinese urbanization. Social Sciences in China, 2012,7, p.82-100.

[5] Deng Xiangzhen, Zhong Haiyue. Path of sustainable urbanization in western China. China Population, Resources and Environment, 2013,23(10) , p.24-30.

[6] Ji Ming, Zeng Weiwei. The regional characteristics of the population flow in China and its Influence on the urbanization. Journal of Guangxi Teachers Education University(Natural Science Edition), 2016,32(2), p.1-9.

[7] Wu Tingfang, Xia Gang. Regional difference, enterprise investment and quality of economic growth. Economic Research Journal, 201492), p.185-189.

[8] Ma Wei, Bai Yongping, Zhang Yonghua. Spatial pattern and convergence of new urbanization in China: 2002-2011. Economic Geography, 2015,35(2) , p.62-72. 
[9] Niu Pinyi, Lu Yuqi. Spatial econometric analysis of regional economic concentration and converage in Jiangsu province. Human Geography, 2013(1) , p.94 - 99.

[10]Chen Bowen, Bai Yongping,Wu Changyan. Research on spatial patterns and potentiality of regional economy in the economic region of Hohhot-Baotou-Erdos-Yulin based on spatial-temporal proximity. Economic Geography,2011,33(1), p.27 - 34.

[11]Jiang Wei. The spatial econometrical analysis to influencing factors of Chinese regional urbanization level,Economic Geography ,2009,29(4) , p.613 - 616.

[12]Zhang Kaiguang, Ba Mingting, Sun Yanming. Study on spatial distribution characteristics of Intra-City agriculture sub-sector value-added growth rates in Henan Province.Advances in engineering research, 2015,33, p.167-171.

[13]Zhang Kaiguang, Meng Hongling, Ba Mingting. The research of algorithm of optimum route calvulation in express traffic system based on time measure. Applied Mechanics and materials,570(3) , p.807-811.

[14]Zhang Kaiguang, Meng Hongling, Ba Mingting. Non-Euclidan algorithm on optimum route planning in urban multi-layer communication.Science of Surveying and Mapping,2014,39(6), p.86-89. 